\title{
Vol. 160 No.1/2 1982
}

\section{F. Théobald, J. Morvan}

Crystal and molecular structure of dimethyl 2,3-dihydro-2,3dihydroxy-4,7-dimethyl-benzo[b]furan-2,3-dicarboxylate 1

\section{S. Hansen, S. Andersson, L. Fälth}

A description of the feldspar framework 9

\section{T. Ishida, Y. Watanabe}

A criterion method for indexing unknown powder diffraction patterns 19

P. García Casado, 1. Rasines

Crystal data for the spinels $\mathrm{MGa}_{2} \mathrm{O}_{4}(\mathrm{M}=\mathrm{Mg}, \mathrm{Mn}) \quad \mathbf{3 3}$

\section{E. F. Paulus, S. Grabley}

Molecular and crystal structure of racemic phosphinothricin hydrate (3-ammonio-3-carboxy-propyl) methylphosphinate-hydrate
P. G. Jones, G. M. Sheldrick, A. J. Kirby, C. M. Evans, R. Glenn, J.Stegemann

The crystal structure of $1-\left(2^{\prime}, 4^{\prime}\right.$ - Dinitrophenoxy $)$-bicyclo[3.3.1]-9oxanonane 45

B. G. Hyde, T. J. White, M. O'Keeffe, A. W. S. Johnson Structures related to those of spinel and the $\beta$-phase, and a possible mechanism for the transformation olivine $\longleftrightarrow$ spinel 53

E. F. Paulus, S. Grabley Molecular and crystal structure of L-phosphinothricin (2-ammonio-4methylphosphinico-butyrate) $\quad 63$

J. Jaud, M. Benhammou, J. P. Majoral, J. Navech Synthèse, structure cristalline et moléculaire du trimethyl-4,6,9 hexaza-1,3,4,6,7,9 phospha-5 tricyclo $\left[3.3 .1 .1^{3.7}\right.$ ] decane 69

\section{E. Zobetz}

Über die Gestalt von $\mathrm{BO}_{3}$-Gruppen

H. H. Otto

Zur Kristallchemie metastabiler Bleigermanate im Konzentrationsbereich $\mathrm{PbO} / \mathrm{GeO}_{2}=5 / 3$ bis $3 / 2$ 
T. Balić-Žunič, S. Śćavničar, P. Engel

The crystal structure of rebulite, $\mathrm{Tl}_{5} \mathrm{Sb}_{5} \mathrm{As}_{8} \mathrm{~S}_{22} \quad 109$

M. Jansen

Zur Struktur von Kaliumdisilicat 127

J. Liebertz, S. Stähr

Zur Existenz und Einkristalizüchtung von $\mathrm{Zn}_{3} \mathrm{BPO}_{7}$ und $\mathrm{Mg}_{3} \mathrm{BPO}_{7}$ 135

E. Kendi

Molecular and crystal structure of tris(hydroxymethyl)aminomethane 139

\section{Short Communications}

K. Peters, E.-M. Peters, H. G. von Schnering, W. Adam,

H. Rebollo

Crystal structure of 4-phenyl-2,4,6-triazatetracyclo- $\left[5 \cdot 3 \cdot 2 \cdot 0^{2.6} \cdot 0^{8.10}\right]$ dodec-11-ene-3,5-dione 145

K. Peters, E.-M. Peters, H. G. von Schnering, W. Adam,

H. Rebollo

Crystal structure of 4-phenyl-2,4,6-triazatricyclo [5.3.2.0 $\left.0^{2,6}\right]$ dodeca-

9,11-dien-3,5,8-trione 149

A. Kirfel, K. Petrov, St. Karagiozova

Crystal data for $\mathrm{K}_{3} \mathrm{FeMO}_{4} \mathrm{O}_{15} \quad 153$

Y. Billiet, H. Burzlaff, H. Zimmermann

Comment on the paper of $\mathrm{H}$. Burzlaff and $\mathrm{H}$. Zimmermann "On the choice of origin in the description of space groups" 155 


\section{Vol. 160 No. $3 / 41982$}

T. Tagai, H. Ried, W. Joswig, M. Korekawa

Kristallographische Untersuchungen eines Petalits mittels

Neutronenbeugung und Transmissionselektronenmikroskopie 159

L. M. Alte da Veiga, L. R. Andrade, W. Gonschorek

The crystal structure of manganese difluoride $\left(\mathrm{MnF}_{2}\right)$ : Reliability test

of hypothetical intensity variances by means of $\chi^{2}$ distributions 171

K.-F. Hesse, F. Seifert

Site occupancy refinement of osumilite $\mathbf{1 7 9}$

W. Gonschorek

$X$-ray charge density study of rutile $\left(\mathrm{TiO}_{2}\right) \quad 187$

C. Chieh

Tautoeikonic sets in some $p, q$ cyclotomies 205

J. K. Mohana Rao, R. K. Rajaram

Crystal structure of ferroelectric tetramethyl ammonium

trichloromercurate $\mathbf{2 1 9}$

R. K. Rajaram, J.K. Mohana Rao

Crystal structure of lead acetate trihydrate $\mathbf{2 2 5}$

E. F. Paulus

Molecular and crystal structure of C.I. Pigment Red 208, 12514, n-Butyl2 - :2 - oxo - 3 - [N - (2 - oxo - 2,3 - dihydro - 5 - benzimidazolyl) - carbamoyl]-naphthylidenhydrazino;-benzoat (PV-Rot HF2B) 235

W. Clegg

A random reflexion search method in four-circle diffractometry $\mathbf{2 4 5}$

R. Enjalbert, G. Guerch, J.-F. Labarre, J. Galy

Crystal structure of $\mathrm{N}_{3} \mathrm{P}_{3} \mathrm{Cl}_{4}\left[\mathrm{HN}\left(\mathrm{CH}_{2}\right)_{3} \mathrm{NH}\right]$ at $133 \mathrm{~K}$ : a spiro cyclic cyclotriphosphazene $\mathbf{2 4 9}$

P. G. Jones, G. M. Sheldrick, A. J. Kirby, R. Glenn

Crystal structure of phenyl- $\alpha$-D-glucopyranoside 259

P. G. Jones, G. M. Sheldrick, W. Clegg, A. J. Kirby, R. Glenn

Crystal structure of phenyl- $\beta$-D-glucopyranoside dihydrate 269 
P. RogI, B. Kunsch, P. Ettmayer, H. Nowotny, W. Steurer

A neutron diffraction study of $\mathrm{Cr}_{3}\left({ }^{11} \mathrm{~B}_{0.44} \mathrm{C}_{0.56}\right) \mathrm{C}_{0.85}$ and $\mathrm{Cr}_{3} \mathrm{C}\left(\mathrm{C}_{0.52} \mathrm{~N}_{0.48}\right) \quad 275$

K.-F. Tebbe, Th. Heinlein

Beiträge zur Strukturchemie phosphorhaltiger Ketten und Ringe.

VI. Die Molekül- und Kristallstruktur des 2,3,4,6-Tetra-tert-

butylbicyclo[3.1.0] hexaphosphans, $t-\mathrm{Bu}_{4} \mathrm{P}_{6} \quad 285$

T. Murakami, Y. Takéuchi, T. Yamanaka

The transition of orthoenstatite to protoenstatite and the structure at $1080^{\circ} \mathrm{C} \quad 299$

Short Communication

L. Fälth, S. Andersson

Crystal structure of the synthetic zeolite $\mathrm{N}, \mathrm{NaAISiO}_{4} \cdot 1.35 \mathrm{H}_{2} \mathrm{O}$ 